\title{
MIRADA DE LOS DOCENTES ANTE LAS PROBLEMÁTICAS ESTUDIANTILES EN EL CICLO BÁSICO DE LA CARRERA MEDICINA VETERINARIA
}

Curso de Acción para la Investigación y el Desarrollo $(\mathrm{CAl}+\mathrm{D})$ : Análisis curricular del ciclo básico de la carrera de Medicina Veterinaria: una mirada integral.

FASCENDINI, Paola Y.'; CIGNETTI, Luciana M.2; GRAMAGLIA, Carina V. ${ }^{3}$; GALVÁN, Stella M. ${ }^{4}$.

${ }^{1}$ Servicio Orientación Educativa. ${ }^{2}$ Idioma Extranjero: Inglés. ${ }^{3}$ Informática. ${ }^{4}$ Anatomía II. Facultad de Ciencias Veterinarias. Universidad Nacional del Litoral. Esperanza, Santa Fe. Argentina. 3080. (54) 03496-420639.pfascendini@fcv.unl.edu.ar

Facultad de Ciencias Veterinarias. Universidad Nacional del Litoral. Esperanza, Santa Fe. Argentina. 3080. (54) 03496-420639.pfascendini@fcv.unl.edu.ar 


\section{MIRADA DE LOS DOCENTES ANTE LAS PROBLEMÁTICAS ESTUDIANTILES EN EL CICLO BÁSICO DE LA CARRERA MEDICINA VETERINARIA}

Curso de Acción para la Investigación y el Desarrollo (CAl+D): Análisis curricular del ciclo básico de la carrera de Medicina Veterinaria: una mirada integral.

FASCENDINI, Paola Y. ${ }^{\text {; }}$ GRAMAGLIA, Carina V. ${ }^{2}$; CIGNETTI, Luciana M. 3; GALVÁN, Stella M. ${ }^{4}$.

${ }^{1}$ Servicio Orientación Educativa. ${ }^{2}$ Informática. ${ }^{3}$ Idioma Extranjero: Inglés. ${ }^{4}$ Anatomía II.

Facultad de Ciencias Veterinarias. Universidad Nacional del Litoral. Esperanza, Santa Fe. Argentina. 3080. (54) 03496-420639. pfascendini@fcv.unl.edu.ar

Facultad de Ciencias Veterinarias. Universidad Nacional del Litoral. Esperanza, Santa Fe. Argentina. 3080. (54) 03496-420639. pfascendini@fcv.unl.edu.ar

\section{Resumen}

El objetivo del presente trabajo es socializar la mirada docente en torno a las problemáticas detectadas en la población estudiantil del ciclo básico de la carrera Medicina Veterinaria, a partir del análisis de los factores que desde su perspectiva inciden en el recorrido académico de los estudiantes. La instrumentación de una encuesta diseñada para docentes permitió acercarnos a las interpretaciones que realizan de lo que acontece en este tramo de la carrera, con el fin de tomar conciencia de aquellos factores que tienden a repercutir significativamente en el desempeño de los jóvenes. Los resultados obtenidos nos indican que los docentes observan dificultades vinculadas a la interpretación de las consignas, las cuales son atribuidas principalmente a la escasa preparación que han obtenido en el nivel secundario, así como también a factores emocionales que tienden a impactar en la continuidad de los estudios. A su vez, expresan que la desorientación vocacional resulta ser un signo importante que repercute en la motivación por la carrera elegida. Otros indicadores importantes refieren a la falta de métodos de estudios propios, y a dificultades para la expresión oral y escrita. Además, señalan que las propuestas pedagógicas ofrecidas no siempre satisfacen las necesidades de los estudiantes actuales.

Palabras claves: problemáticas estudiantiles, mirada docente, ciclo básico, Medicina Veterinaria. 


\section{Summary}

The objective of this paper is to socialize teachers' view about problems detected among students of the basic cycle of the Veterinary Medicine course on the basis of the analysis of the factors that from their perspective influence the students' academic path. The implementation of a survey designed for the teachers allowed us to get closer to the interpretations they make of what happens along this stretch of the course, in order to become aware of those factors that tend to have a significant impact on the students' performance. The results obtained indicate that the teachers observe difficulties related to the students' interpretation of instructions, which are mainly attributed to the poor preparation they obtained at secondary school, as well as emotional factors that tend to impact on their continuity of studies. At the same time, they express that vocational disorientation turns out to be an important sign, which affects the motivation for the course of studies chosen. Other important indicators refer to the lack of self-study methods, and difficulties for oral and written expression. It should be noted that the pedagogical proposals offered do not always fulfills the real students' needs.

\section{Keywords:}

student problems, teaching outlook, basic cycle, Veterinary Medicine.

\section{Introducción}

En la carrera Medicina Veterinaria de la Facultad de Ciencias Veterinarias dependiente de la Universidad Nacional del Litoral se evidencia la influencia del enciclopedismo, y las clases expositivas reflejan las líneas de abordaje curricular sobre las que se encuentra asentada la enseñanza de algunas prácticas educativas. Algunas experiencias significativas de capacitación han surgido en torno a intentar modificar los procesos de enseñanza y de aprendizaje, las que han permitido renovar y modificar progresivamente algunas de las prácticas docentes, pero aún sin sistematizarlas de manera completa, ya que en muchos casos predomina la duda y la inquietud en torno a la aplicación de los nuevos paradigmas educativos.

En este contexto, urge priorizar al estudiante como eje central del proceso educativo y su condición de aprendiz, ya que la tarea primordial es favorecer los procesos de cada cual en la búsqueda de aprendizajes significativos y autorregulados. En esta oportunidad, la mirada está centrada en los docentes, quienes con su visión y misión intentan observar lo que acontece alrededor, tomando conciencia de la implicancia de 
su rol en esta realidad, pero también conscientes de su falta de herramientas en cuanto a una formación que permita andamiar los procesos desde otra mirada más integral y procedimental, lo cual ellos mismos han verbalizado.

En el presente trabajo compartimos el análisis, resultados e interpretación de una encuesta diseñada por docentes-investigadores integrantes del proyecto Curso de Acción para la Investigación y el Desarrollo $(\mathrm{CAI}+\mathrm{D})$ titulado "Análisis curricular del ciclo básico de la carrera de Medicina Veterinaria: una mirada integral”, cuyo propósito esencial es detectar y atender a las dificultades que los estudiantes atraviesan en el ciclo básico de la carrera.

\section{Metodología}

El diseño metodológico corresponde a un estudio descriptivo, y el análisis de los datos recolectados se realizó bajo un enfoque cuantitativo. La herramienta utilizada para la recolección de datos fue una encuesta estructurada sobre la base de ejes centrales. Estos ejes se relacionan con la mirada del docente sobre los factores que inciden en el recorrido académico que los estudiantes realizan, en particular, las dificultades que ellos observan que los estudiantes presentan en los proceso de ingreso y permanencia en la universidad y en su desempeño académico, así como también las estrategias metodológicas y modalidades de clase utilizadas para propiciar el aprendizaje, los formatos de evaluación y los aspectos ponderados durante los procesos evaluativos. Para la aplicación de la encuesta, se facilitó a los docentes un enlace a un formulario en Google Drive, en el que se consultó acerca de estos aspectos. Esta herramienta permite analizar los datos y representarlos de manera gráfica y visual. La misma fue respondida por 33 de un total de 69 docentes que dictan clases en las asignaturas del ciclo básico de la carrera.

\section{Resultados}

En relación con las dificultades que afectan la adaptación estudiantil, los resultados indican que el $62,5 \%$ de los docentes los vincula con compromisos emocionales; el $56,3 \%$, con la falta de motivación en la carrera; el $50 \%$, con la desorientación vocacional, y el $28,1 \%$, con factores económicos (Gráfico 1). 


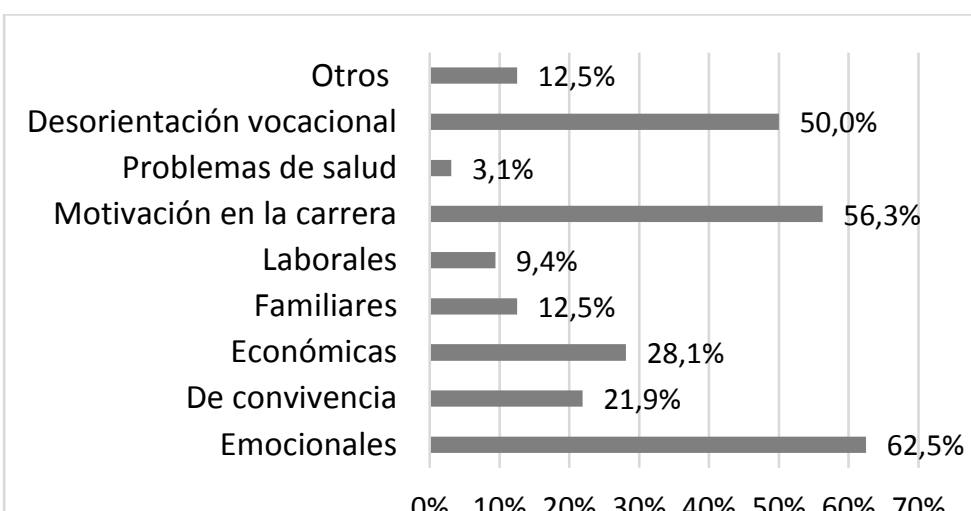

Gráfico 1. Dificultades que afectan la adaptación de los estudiantes al sistema universitario.

Con respecto a la evaluación que los docentes hacen de las dificultades más recurrentes en el desempeño académico de los estudiantes ingresantes de la cohorte 2017 , el $72,7 \%$ de los docentes percibe que los mismos presentan dificultades en la expresión oral y en la comprensión, el $66,7 \%$ cree que el problema reside en la ausencia de métodos de comprensión, el $54,5 \%$, en la falta de métodos de estudio, y el $57,6 \%$, en su débil formación en el nivel secundario (Gráfico 2).

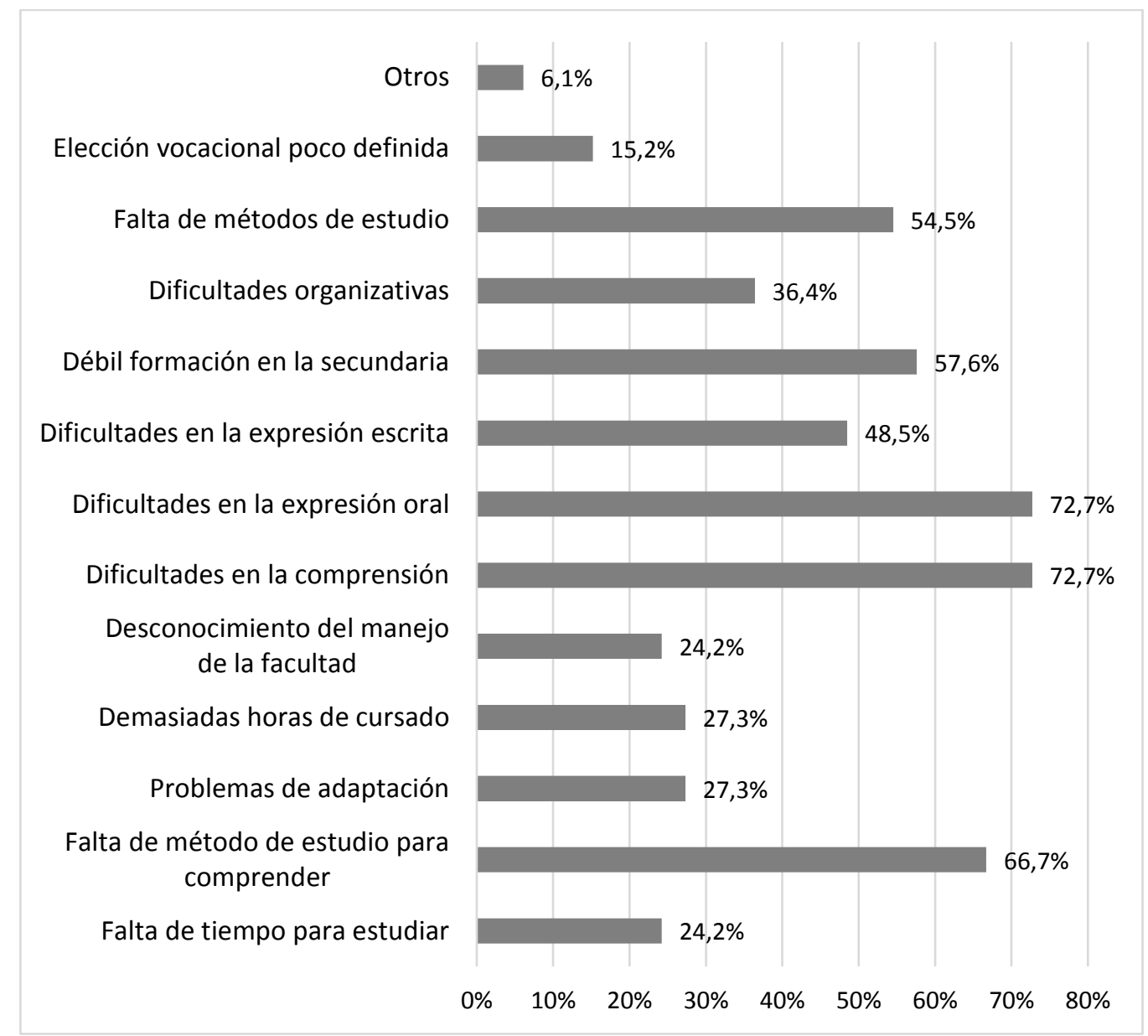

Gráfico 2. Dificultades que inciden en el desempeño académico. 
Otro de los factores analizados es el uso de estrategias metodológicas propicias para favorecer el aprendizaje de los estudiantes, entre las cuales el 90,9\% de los docentes mencionaron la utilización de ejemplos como un modo de vincular teoría con práctica, aplicación de contenidos a situaciones problemáticas cotidianas, y 84,8\% expresaron integración de contenidos nuevos con los previos (Gráfico 3).

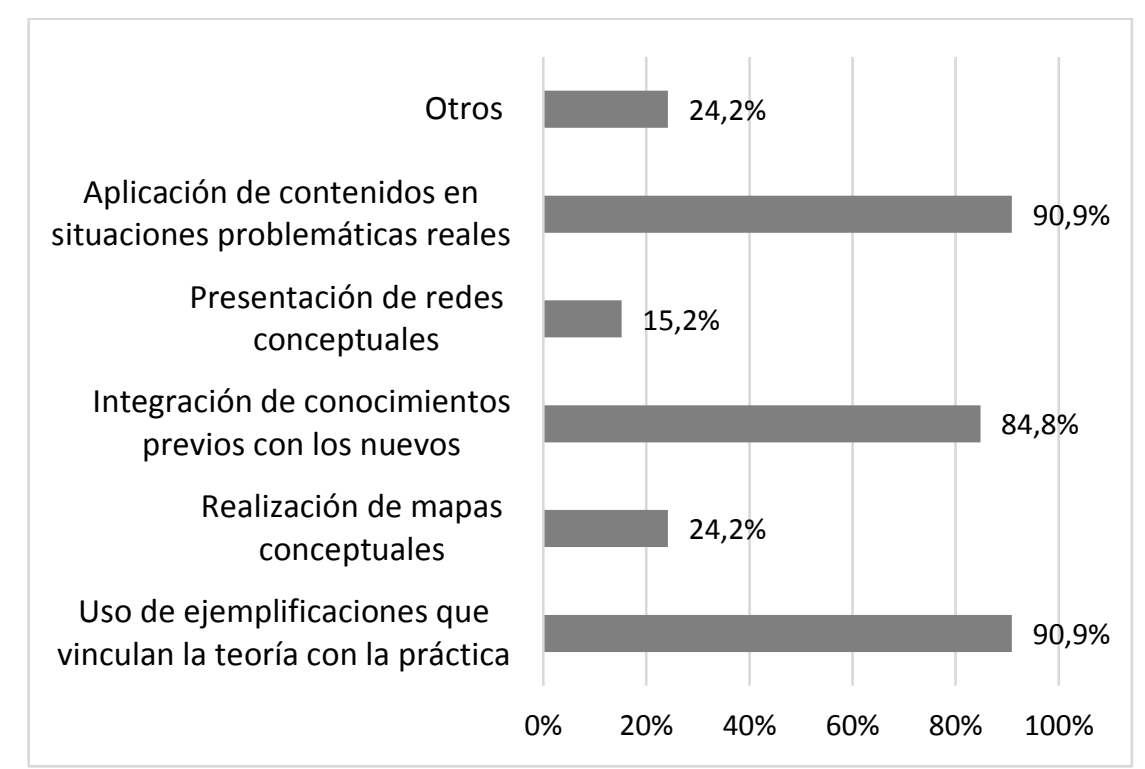

Gráfico 3. Estrategias metodológicas que utilizan los docentes para favorecer el aprendizaje.

En relación con las modalidades que predominan en el dictado de las clases, el 93,9\% sostiene combinar clases prácticas y teóricas; el 72,7\%, utilizar recursos digitales; el $63,6 \%$, relacionar los temas; el 60,6\%, participar al alumnado, y el 33,3\%, llevar a cabo prácticas dinámicas e interactivas (Gráfico 4).

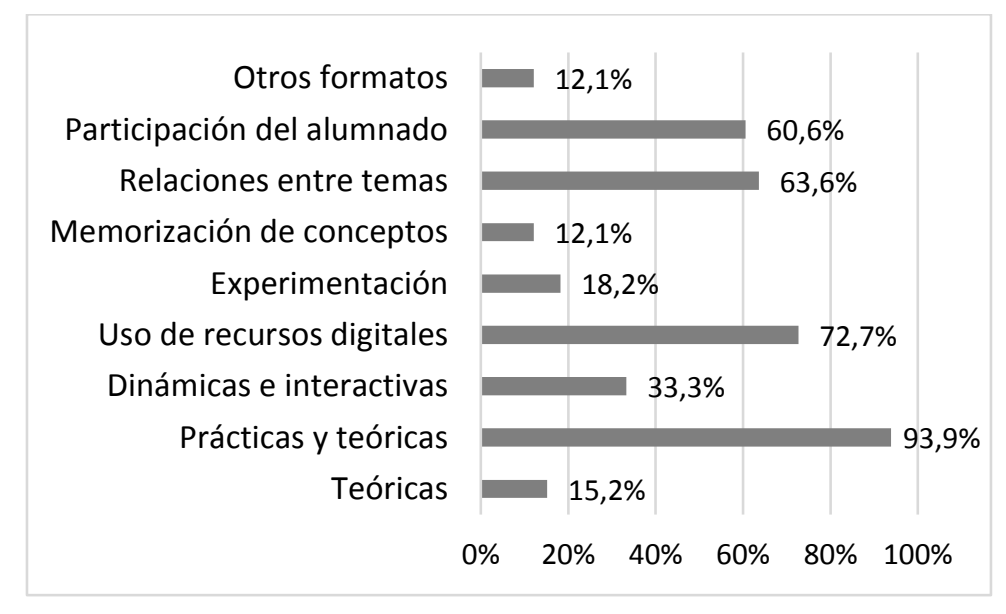

Gráfico 4. Modalidades que predominan en el dictado de clases.

Con respecto a los formatos evaluativos instrumentados, se hace referencia al uso de exámenes múltiple opción por parte del $54,5 \%$ de los docentes; el $51,5 \%$ sostiene 
instrumentar exámenes orales; el 42,4\%, trabajos grupales; el 36,4\%, exámenes verdadero / falso, y el 30,3\%, exámenes de desarrollo (Gráfico 5).

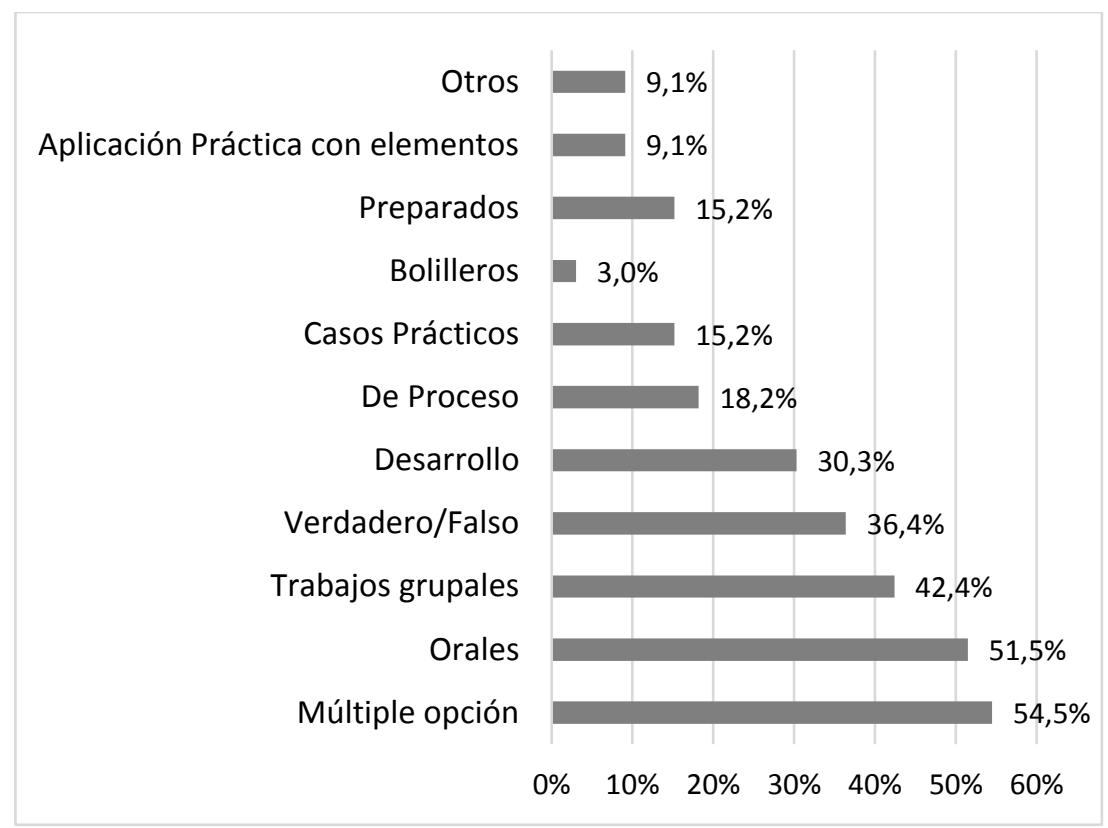

Gráfico 5. Formatos evaluativos instrumentados.

Al momento de ponderar el desempeño de los estudiantes: el 81,8\% tiene en cuenta el porcentaje de asistencia y la calificación obtenida en las evaluaciones parciales; el $45,5 \%$, la fluidez verbal y la participación; el $42,4 \%$, las habilidades procedimentales, y el 36,4\%, la forma de expresarse por escrito (Gráfico 6).

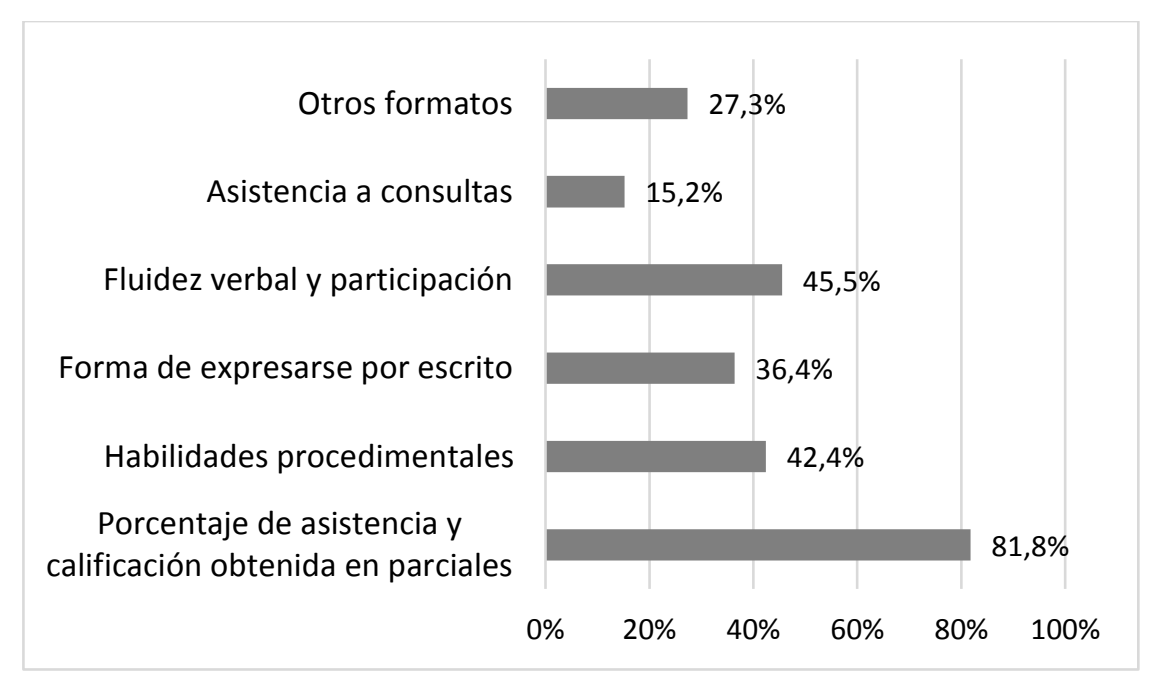

Gráfico 6. Aspectos considerados al momento de ponderar el desempeño de los estudiantes. 
La falencia más significativa identificada con relación a cuestiones evaluativas ha sido la interpretación errónea de consignas, la cual fue señalada por el $75,9 \%$ de los docentes (Gráfico 7).

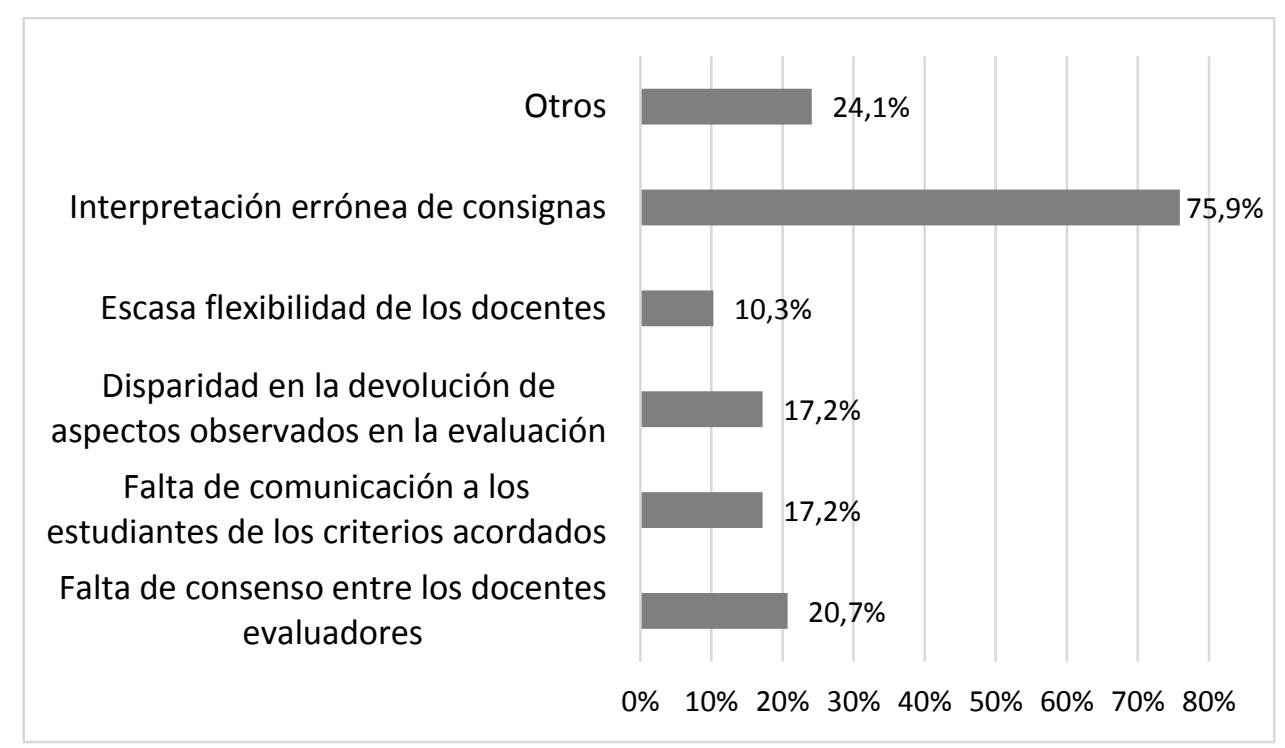

Gráfico 7. Dificultades observadas en relación con las instancias de evaluación.

En relación con los aspectos que según los docentes impactan al momento de rendir una materia, se mencionaron los siguientes: el $87,9 \%$ considera la utilización del material bibliográfico de la cátedra como primordial; el 81,8\% además menciona la interiorización en los criterios evaluativos, y el 72,7\%, en la modalidad de examen. El $72,7 \%$ de los docentes también considera indispensable la accesibilidad a las consultas; el 66,7\% considera como un factor relevante el horario en que se realiza el examen, y el $57,6 \%$, que los estudiantes conozcan la planificación de la asignatura (Gráfico 8).

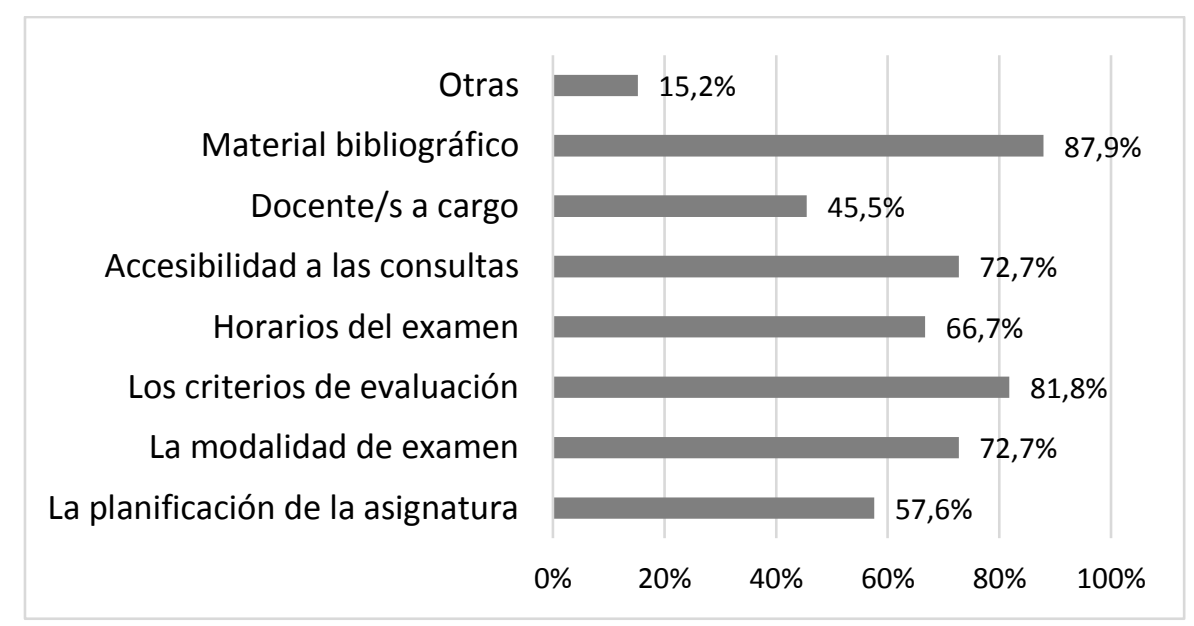

Gráfico 8. Aspectos que impactan en el desempeño de los estudiantes en las evaluaciones. 


\section{Discusión}

En el análisis que los docentes hacen acerca de las dificultades más recurrentes que afectan la adaptación de los estudiantes ingresantes cohorte 2017 se pudo observar que las mismas parecen relacionarse con motivos multifactoriales, con lo cual resulta verdaderamente difícil abordar la problemática desde un solo lugar. Es decir, se debe realizar un abordaje integral que contribuya a que los estudiantes afronten y superen estas dificultades e, idealmente, que alcancen la permanencia en nuestra institución en el tiempo estipulado hasta alcanzar el objetivo deseado.

En cuanto a la percepción de los docentes sobre las dificultades más recurrentes en el desempeño académico de los estudiantes, se destacó la falta de competencia para la expresión oral, lo cual se puede relacionar con un aprendizaje memorístico que tiende a limitar la expresión como resultado del posicionamiento pasivo que se genera ante el material de estudio, ante ello, una consideración que tendrían que tomar los docentes es apuntar al desarrollo de procesos de construcción activa del conocimiento y, a su vez, de habilidades comunicativas para la expresión (en este caso en el medio oral) de ese conocimiento construido.

Además, los docentes señalan que otra de las debilidades de estos estudiantes es la falta de comprensión e interpretación, y aquí se plantea un problema detonante para la enseñanza universitaria, sobre todo teniendo en cuenta que el modo escrito sigue siendo predominante a la hora de acceder al conocimiento. La enseñanza de la lectura es una tarea compleja ya que implica que el estudiante desarrolle tres habilidades: entender, comprender e interpretar. Entender implica captar los significados literales de las palabras del texto, asociándolas con significados semánticos para poder reconstruir las ideas codificadas por el emisor en el mensaje. Por su parte, la comprensión implica en primer lugar, decodificar el texto y, a su vez, captar el significado, el sentido y el contenido complementario, conectando de manera coherente los conocimientos previos con la información nueva que aporta el texto. Por último, interpretar un texto tiene una complejidad mayor a la de entenderlo o comprenderlo ya que implica que el discente/lector establezca el espacio sociocultural, la significación inferencial, sepa crear imágenes y ubicar el macro-contexto (Almaguer Luaiza \& Peña González, 2009). Esto se podría relacionar con el otro inconveniente advertido por los docentes, que es la débil formación en el nivel secundario. Consideramos que es crucial que el desarrollo de estas habilidades (las cuales son variables) alcancen su máximo potencial en este nivel educativo para favorecer el ingreso de los estudiantes al contexto universitario. 
En cuanto a lo metodológico, los docentes manifestaron utilizar una amplia variedad de estrategias propicias para favorecer el aprendizaje de los estudiantes. Sin embargo, consideramos fundamental que en cada espacio curricular se pongan en práctica metodologías de enseñanza que tengan en cuenta las didácticas específicas para la enseñanza de cada área disciplinar en particular. En este sentido, la metodología situada sobre la formulación de interrogantes y planteamiento de problemas permite atravesar el conocimiento desde la interdisciplinariedad, sin que ello implique la renuncia por lo disciplinar, ya que lo verdaderamente importante es buscar preguntas relevantes que permitan suscitar el interés estudiantil y la movilidad por conocer. Cabe señalar que cualquier proceso de aprendizaje solo es realmente eficaz cuando nos enfrentamos a problemas reales e intentamos resolverlos con esfuerzo (Carbonell, 2009). Es a partir de la propia experiencia cuando sabemos cómo resolver dificultades, cobrando sentido el aprendizaje tanto propio como de los otros, debido a que con el paso del tiempo sólo permanece aquello que nos ha preocupado, nos ha hecho pensar y nos ha motivado para buscar respuestas razonables (Carbonell, 2009).

A su vez, y en relación con el dictado de las clases, los docentes también admiten utilizar una variada gama de modalidades. Aún así, es necesario que el estudiante adquiera un mayor nivel de protagonismo en las clases, desarrollando una visión del mundo desde su propia perspectiva a través de un proceso de aprendizaje en acción. Desde este enfoque, se espera que el docente desempeñe el papel de tutor y facilitador, al orientar al estudiante, retroalimentar su aprendizaje y complementarlo con actividades metacognitivas que estimulen la auto-conciencia sobre el proceso de pensamiento (Davini, 2008). Finkel (2008) consideraba a la narración como una estrategia ineficaz para enseñar, aunque sea el instrumento mayormente empleado. De este modo se pone énfasis en el aprendizaje como el factor clave de la educación para desarrollar espacios y aulas donde el estudiante pueda aprender desde un lugar crítico, autorregulado y participativo, involucrándolo en los procesos formativos. Desde esta perspectiva existe una convicción de que el conocimiento y el proceso de aprendizaje no descansan en una autoridad exclusivamente, sino en nuestra propia mente, y el papel del docente es estar preparado para lo imprevisible, siendo faros para evitar que se pierdan comentarios y aportaciones de mayor interés, formulando preguntas que despierten la indagación, consiguiendo que la conversación ocurra y trascienda, y resumiendo los puntos clave del intercambio (Finkel, 2008).

Con respecto a los formatos evaluativos instrumentados y a la valoración del desempeño de los estudiantes, los docentes expresaron utilizar diferentes formatos y 
considerar distintos aspectos a la hora de evaluar a los estudiantes. Consideramos necesario resaltar que cada instrumento de evaluación debe ser coherente con los objetivos y con el objeto de evaluación. Además, es indispensable que el alumno no sólo tenga acceso a la enseñanza de todos los contenidos, sino que también sepa cómo va a ser evaluado y los criterios de ponderación del docente y su equivalente en términos de calificaciones. A su vez, debe contar con una instancia de retroalimentación que le permita reorientar su aprendizaje. En este sentido, resulta deseable la incorporación de evaluaciones formativas (de proceso) como complemento de las evaluaciones sumativas (de resultados). "Mediante la evaluación formativa se intenta utilizar el conocimiento para reconducir y hacer progresar las intervenciones y ayudar a los participantes a conseguir sus objetivos, de tal forma que se produzca una relación recíproca entre los evaluadores y los responsables de la acción educativa. La evaluación formativa exige, obviamente, que sea desarrollada en el decurso de la acción, estableciéndose un proceso de interacción permanente entre la evaluación y la acción" (Fernández Sierra, 1994, p. 12).

\section{Conclusión}

Podemos concluir que uno los fines últimos en el ámbito de la educación es que los discentes logren aprendizajes profundos y duraderos, los cuales son fundamentales para que se conviertan en buenos profesionales. Por su parte, los docentes tienen el desafío de fomentar este tipo de aprendizajes en los estudiantes, promoviendo su participación en el proceso de construcción de su propio conocimiento. Las tradiciones arraigadas en la enseñanza imprimen un modelo natural de impartir clase a partir de la narración, precisamente porque el aprendizaje se comprende más como una absorción de una información específica brindada por un discurso docente elocuente que le da autoridad y poder. Por lo cual, es necesario cuestionar los modelos sobre los que se asienta la enseñanza. Desde esta perspectiva, se requiere problematizar las propias creencias acerca de la misma (Finkel, 2008). Un buen docente se encuentra conectado con los aprendizajes de terceras personas, en los que se incluyen formatos variados que permitan que todos aprendan y la evaluación es considerada como una instancia de crecimiento y de formación. 


\section{Referencias}

- Almaguer L., C. B \& Peña González, R. M. (2009). La lectura y las diferencias entre entender, comprender e interpretar. Recuperado de:

https://aal.idoneos.com/revista/ano 11 nro. 13/entender, comprender e interpreta $\underline{r}$

- Carbonell, J. (2009). Dar clase con la boca cerrada. Revista electrónica sobre la enseñanza de la economía pública, 6, 49-60.

- Davini, C. (2008). Métodos de enseñanza. Didáctica general para maestros y profesores. Argentina, Buenos Aires: Santillana.

- Fernández Sierra, J. (1994). Evaluación del Currículum, perspectivas curriculares y enfoques en su evaluación. En J. Angulo Rasco y N. Blanco (Coord.), Teoría y desarrollo del currículum (pp. 297-312). Málaga: Aljibe.

- Finkel, D. (2008). Dar clase con la boca cerrada. Traducción de Óscar Barberá. Valencia: Publicacions de la Universitat de Valéncia (1 ${ }^{\text {a }}$ ed. inglesa, 2000). 